\title{
El Corno Emplumado y la vanguardia en el umbral de la nueva
} era ${ }^{1}$.

\section{El Corno Emplumado and the avant-garde on the threshold of the new era.}

DOI: $10.32870 /$ sincronia.axxv.n80.18b21

\author{
Alberto Rodríguez González \\ Universidad Autónoma Metropolitana- Azcapotzalco (MÉXICO) \\ CE: argon107@hotmail.com / ORCID: 0000-0002-2899-3740
}

Esta obra está bajo una Licencia Creative Commons Atribución-NoComercial 4.0 Internacional

Recibido: 31/03/2021

Revisado: $14 / 05 / 2021$

Aprobado: 09/06/2021

\section{RESUMEN}

Este artículo aborda el caso de la revista mexicana El Corno Emplumado y su papel como una publicación de vanguardia desde la idea de movimiento de vanguardia de Renato Poggioli y las nociones de umbral de época y estética de umbral formuladas por Hans Robert Jauss y Luciana del Gizzo, respectivamente. La intención es examinar la manera en que la revista editada por Margaret Randall y Sergio Mondragón actualiza el impulso utópico de las vanguardias a partir de su visión de que el cambio social a inicios de los años 60 del siglo XX habría de llegar gracias a la renovación espiritual que sólo podrían proporcionar el arte y la poesía. Adicionalmente se indaga la manera en que la revista reconfigura el mito vanguardista del nuevo comienzo desde su postulado del advenimiento de una nueva era, la era del hombre de aire. Todo ello con el fin de discutir la posibilidad de pensar en la presencia de una continuidad vanguardista en la literatura mexicana a lo largo del siglo XX.

\footnotetext{
1 El presente artículo forma parte del proyecto de estancia posdoctoral 2020- 2021, en la Maestría en Literatura Mexicana Contemporánea de la Universidad Autónoma Metropolitana - Azcapotzalco, financiada por el Consejo Nacional de Ciencia y Tecnología (CONACYT).
} 
Palabras claves: Revistas literarias mexicanas, El Corno Emplumado, Vanguardias latinoamericanas, Poesía del siglo XX.

\begin{abstract}
This article analyzes the case of the Mexican magazine El Corno Emplumado and its role as an avant-garde publication based on Renato Poggioli's idea of avant-garde movement and the notions of epoch threshold and aesthetics of threshold formulated by Hans Robert Jauss and Luciana del Gizzo, respectively. The intention is to examine the way in which the magazine edited by Margaret Randall and Sergio Mondragón updates the utopian impulse of the avant-gardes based on their vision that social change at the beginning of the $60 \mathrm{~s}$ of the 20th century would come thanks to renewal spirituality that only art and poetry could provide. Additionally, it investigates the way in which the magazine reconfigures the avantgarde myth of the new beginning from its postulate of the advent of a new era, the era of the man of air. All this in order to discuss the possibility of thinking about the presence of an avant-garde continuity in Mexican literature throughout the 20th century.
\end{abstract}

Keywords: Mexican literary magazines, El Corno Emplumado, Latin American Avant-garde, Twentieth century poetry.

El mes de enero de 2022 se cumplirán 60 años de la publicación del primer número de El Corno Emplumado, con una existencia de siete años y 31 números publicados, se trata sin duda de una revista cuyo importante papel en las letras mexicanas contemporáneas no ha sido suficientemente aquilatado. El proyecto editado por la poeta estadounidense Margaret Randall y el mexicano Sergio Mondragón tuvo como eje la convicción de que al inicio de la década de 1960 se hacía necesaria una renovación social, cuya posibilidad descansaba en el arte y desde esa perspectiva asume como su tarea la difusión de poetas y artistas plásticos que apostaran por dicha transformación.

La revista desempeñó un papel fundamental en la promoción de la obra de jóvenes poetas y artistas de México, Latinoamérica y Estados Unidos que desde una actividad personal o colectiva apostaban de la misma manera por una transformación estética. A partir de esta labor, algunos acercamientos críticos consideran a El Corno Emplumado como una expresión de las vanguardias o neovanguardias latinoamericanas con actividad en la década de los años 60 (Galindo, 2013). 
Al hacer un balance retrospectivo sobre los años de existencia de la revista y reflexionar críticamente sobre la posibilidad de hablar de un resurgir de la vanguardia en los años 60, Sergio Mondragón rechaza hablar de una "segunda" vanguardia, como una "reaparición" de aquella que emergió y declinó hacia los años 20 del siglo pasado para regresar en décadas posteriores. Ante la cuestión, el editor y poeta postula la posibilidad de pensar más bien el fenómeno vanguardista como una constante permanente en el arte poético, para lo cual recurre a la fórmula de la tradición de la ruptura y postula como hipótesis:

[...] la existencia de una vanguardia permanente en el arte literario, asociada a una tradición de la ruptura, generadora y recipiente de las novedades y las metamorfosis de las formas escritas, aquello que implica el abandono de las convenciones anteriores, reverberaciones del idioma que en su perenne movilidad nos recuerda siempre que el evento central de la vida y el arte literario es el perpetuo cambio [...] (Mondragón, 2006, s/p)

La afirmación de Mondragón se torna especialmente relevante cuando nos encontramos en la víspera de que la vanguardia mexicana llegué a su centenario -si se toma como referencia la irrupción del estridentismo en diciembre de 1921-. Frente a la postura del poeta y editor, se antoja necesaria una reflexión sobre el uso de la noción de vanguardia para referirse precisamente a productos más contemporáneos, como El Corno Emplumado o el movimiento infrarrealista de los años 70. Al respecto, Julio Premat (2013) advierte que el término vanguardia se ha vuelto semánticamente confuso y que por su excesiva utilización termina siendo más que un denominador pertinente, la "marca comercial de la modernidad" (2007, p. 51). El crítico apunta que usar indiscriminadamente el término vanguardia se reduce a "una manera de trasladar lo desconocido a un terreno conocido y bien delimitado y a una historia literaria que se pretende definitiva" (p. 51).

En este contexto, Premat (2013) afirma que la tarea crítica debería omitir afirmar si tal o cual corriente se inscribe en la vanguardia, puesto que el término mismo no define una categoría literaria y señala por el contrario que la tarea de la crítica debería considerar la noción de 
vanguardia más como un "efecto del que hay que analizar, en cada ocurrencia, el origen y funcionamiento" (p. 52).

Desde esta perspectiva, la intención de este trabajo es plantear una serie de interrogantes alrededor de El Corno Emplumado, tomando como horizonte la noción de vanguardia. En ese sentido, vale la pena preguntarse qué implicaciones tiene la posibilidad de postular una vanguardia permanente, como señala Mondragón y de la cual El Corno Emplumado sería un componente importante: ¿En qué sentido es viable postular que las vanguardias de principios del siglo XX forman parte de una continuidad que se expande a lo largo del siglo? ¿Cuáles son los mecanismos, recursos o epistemes que para los años 60 una revista como el Corno reactiva o transforma a fin de poder identificarla como expresión de vanguardia? ¿Si visualizamos a las vanguardias desde su configuración como una labor colectiva (pensemos en los llamados ismos), es decir, como un movimiento, cómo articula el propio Corno su praxis literaria para configurarse en movimiento? Y finalmente, ¿cómo incide la reactivación del programa vanguardista en el discurso de la revista y en los aspectos formales y temáticos de los textos que acoge y difunde?

Para este fin, la propuesta es abordar primariamente el tema recuperando la noción de estética de umbral que desarrolla Luciana del Gizzo a partir de la idea de umbral de época de Hans Robert Jauss en Las transformaciones de lo moderno (2004). En segunda instancia, aparece el tema de la pertinencia de poder caracterizar a una revista como un colectivo, a la manera de los ismos vanguardistas, para lo cual recurro a la noción de movimiento de vanguardia de Renato Poggioli y la concepción de la vanguardia como un movimiento cultural. Finalmente, se examina el programa de la revista que reconfigura el mito vanguardista del nuevo comienzo de la historia al postular en tono milenarista el advenimiento de la Era del Hombre de Aire como una era de renovación y fraternidad universales.

A la luz de estas nociones, se examinan brevemente los primeros años de la revista El Corno Emplumado/The Plumed Horn, a fin de aportar elementos para identificar ciertos componentes asimilados a las vanguardias que la revista actualiza desde su discursividad. 


\section{Una novedad hecha para durar}

Cuando se habla de vanguardias latinoamericanas, existe entre la crítica un cierto acuerdo tácito en que se trató de un fenómeno cuyo inicio y fin se puede fechar. En este tenor y dependiendo de la fuente, se puede ubicar su aparición hacia el final de la primera década del siglo XX y su posterior extinción en la década del 30. Existe también la idea más o menos general de que ese conjunto de movimientos, revistas y artistas que se asimila a la noción de vanguardias mantenían un carácter común asociado al concepto de lo nuevo. Así, por ejemplo, Hugo J. Verani sintetiza el tema:

En el continente latinoamericano los límites temporales de los vanguardismos son, aproximadamente, 1916 y 1935. Las inquietudes renovadoras canalizan hacia 1922 -año clave de la eclosión vanguardista latinoamericana- en una acelerada sucesión de manifiestos, polémicas, exposiciones y movimientos encaminados por propósitos distintos, pero contagiados de la furia de novedad de que habla Jorge Mañach en su ensayo "Vanguardismo" [...] (1990, p. 11).

Los dos elementos enunciados por Verani: el marco cronológico de la existencia de las vanguardias y la preeminencia de la novedad como su marca de identidad, además de representar un problema desde el punto de vista de un Sergio Mondragón que niega la desaparición de dichas vanguardias, han sido también objeto de debates críticos más recientes, tanto en lo relativo a la idea de la vanguardia como fenómeno claramente datado, así como en el tema de su novedad.

Desde esta perspectiva, la investigadora Luciana del Gizzo se pregunta sobre la posibilidad de que la vanguardia pueda permanecer en el tiempo a lo largo del siglo XX y si desde la crítica es posible aplicar la categoría de "vanguardia" a expresiones artísticas y literarias que surgen décadas después de la época de las llamadas vanguardias históricas.

Del Gizzo formula la idea de que, para una comprensión más amplia de la vanguardia como un objeto integral, hay que considerar que, si las nociones de novedad y de ruptura aparecen como paradigma fundacional en la vanguardia, lo hacen como una discursividad que surge por el hecho 
que sólo en el siglo XX se dan las condiciones de posibilidad que justificarían la factibilidad de tal ruptura con el pasado y poder así construir permanentemente la novedad:

Más claramente, la novedad o la ruptura no son características del arte, sino una discursividad propia de la vanguardia como modalidad estética del siglo XX, que pudo tener lugar en ciertas condiciones de posibilidad de esos enunciados los cuales, como se verá, coinciden con una necesidad histórica de cambio profundo y recomienzo. Sólo en estos términos, que aquí se referirán como estética de umbral, es posible pensar en la vanguardia como categoría estética persistente durante todo el siglo (2015, p. 5).

Para la autora, cuando se piensa que la idea de novedad es central en la vanguardia, la posibilidad de que esa novedad retorne constantemente constituye sin duda un problema cuando se trata de pensar a la vanguardia en su aspecto de novedad absoluta como una constante. Del Gizzo se pronuncia entonces por buscar referentes teóricos que den cuenta de la vanguardia no como un proyecto fragmentado, sino como una categoría con presencia permanente a lo largo del siglo XX. Frente a este problema, la investigadora recurre al concepto de umbral de época que propone Hans Robert Jauss.

En la colección de ensayos que agrupa bajo el título de Las transformaciones de lo moderno, Jauss establece que la modernidad estética se transforma desde su inicio hasta su declive en una serie de momentos que él llama umbrales de época, en los cuales los conceptos y prácticas artísticas se transforman sustancialmente. Precisamente la irrupción de la vanguardia supone para Jauss uno de estos umbrales de época, el cual cifra en el año de 1912 (2004, p.14) y estaría conformado por dos oleadas, la primera surge antes de la Primera Guerra Mundial y la segunda después del conflicto armado. En la primera oleada incluye al futurismo (italiano y ruso), el dadaísmo, el expresionismo y el constructivismo, mientras que en la segunda oleada ubica al surrealismo como su expresión fundamental.

El modelo de Jauss presenta la ventaja de que no entiende a la vanguardia como una llamarada que inicia y concluye en un momento específico y restringido, sino que desde la 
perspectiva de los umbrales de época, se entiende que la vanguardia ocurre en oleadas recurrentes a lo largo del siglo XX como una respuesta a diferentes escenarios históricos específicos y conflictivos, los cuales constituyen la apertura de nuevos horizontes históricos, mismos que sólo pueden resultar plenamente aprehensibles a partir de la experiencia estética:

[...] en el seno de la conciencia, todavía indivisa, de la unidad epocal del siglo XX, se produce una progresiva separación del pasado. Parece que lo nuevo que comienza no es susceptible de ser articulado como experiencia, pero que lo viejo que se separa sí es captable en su figura evanescente. Es precisamente la experiencia estética la que puede iluminar este cambio de horizonte (2004, p. 68-69).

Para Jauss, frente a las profundas transformaciones históricas que se suceden con gran rapidez en el periodo de la modernidad, la experiencia estética que se produce en el momento mismo del cambio, se instaura como el recurso necesario para establecer un proceso hermenéutico frente al cambio de horizonte histórico:

Si creemos al historicismo riguroso cuando mantiene que lo nuevo in eventu acostumbra a sustraerse a la experiencia consciente y que sólo ex eventu, retrospectivamente, es reconocido como el límite entre lo que "ya no es" y lo que "aún no es", ¿no le quedará a la experiencia estética esa oportunidad, siempre confirmada, de apostrofar, frente a la experiencia histórica, la aparición de lo nuevo, de elevar a la conciencia las posibilidades que se anuncian, o incluso de dramatizar, como un nuevo comienzo o como un giro único a la manera del paradigma paulino-cristiano ("mira, todo es nuevo"), ese cambio de horizonte todavía imperceptible? (2004, p. 71)

A partir de estas nociones de Jauss, la investigadora Luciana del Gizzo propone entender a la emergencia de los diferentes grupos de vanguardia, aún aquellos que surgen más allá de los años 20, como la presencia de una práctica constante en el siglo XX. En suma, se trata de entender la vanguardia como una praxis unitaria propia de la modernidad en su etapa postrera. Así, los 
diferentes grupos o expresiones de vanguardia se articulan a través de una experiencia estética de umbral, por ello, señala Del Gizzo:

Toda vanguardia es un momento de inflexión en el devenir artístico que sustancia lo que ya no es y lo que todavía no es, señalando de ese modo las cesuras históricas; en ese paréntesis despliega su experimentación vacilante, inexacta e incompleta, que conforma su estética de umbral (2015, p. 54).

Por otro lado, Del Gizzo concibe a los grupos de vanguardia como objetos culturales con el poder para detener el devenir temporal y abrir el tiempo a visiones alternativas desde la experiencia estética que proponen. Apoyándose en ideas de Susan Buck-Morss, Del Gizzo propone que la experiencia estética que postula la vanguardia tiene la potencialidad para sustraer al individuo de la complacencia moral y la resignación política (2015, p. 54).

Al colocarse en sus respectivos umbrales de época, los objetos culturales que son las vanguardias tienen ante sí la tarea de denunciar aquello que ya no es y, simultáneamente, en un afán utópico, proponer aquello que aún no tiene forma. La propia Del Gizzo advierte que tal tarea adquiere para el artista de vanguardia los visos de una lucha con el ángel, ante la cual se hace indispensable construir estrategias específicas y triunfar así en la empresa. Es así entonces que el recurso más eficaz frente a la ciclópea empresa vanguardista será la acción colectiva, pues es en el grupo y no en la individualidad, donde las vanguardias adquieren su fortaleza y capacidad de acción.

Ubicadas en este umbral de cambio, las vanguardias proponen una transformación profunda que desata una respuesta violenta por parte de un entorno artístico que recela de sus ímpetus renovadores. Frente a la hostilidad, la vanguardia enfrenta la necesidad de legitimar sus propias iniciativas que no concuerdan con el paradigma dominante. En este entorno, la colectividad vanguardista cumple la función de dar legitimidad y coherencia a la experimentación que emprenden los integrantes del colectivo y a las cuales se les niega la calidad de artísticas, por ello, apunta Del Gizzo: 
Si la vanguardia pone a prueba lo que se considera arte en una época dada, únicamente puede hacerlo porque hay una instancia que, basada en su carácter colectivo, valida como tal eso que no es considerado artístico por las instancias tradicionales de legitimación. La aceptación de la experimentación individual sería improbable o tendría resistencia y una mayor probabilidad de fracaso. El grupo funciona como garante hacia afuera y hacia su interior convalida prácticas (2015, p. 56).

Así pues, es viable pensar que la eclosión de las vanguardias corresponde a una de las transformaciones que la modernidad experimenta de manera constante en el siglo XX, la cual se inserta en un umbral de época determinado. Tenemos por otro lado, que las prácticas vanguardistas son acciones eminentemente grupales, toda vez que la labor de grupo funciona como un mecanismo de auto legitimación de la estética de umbral propia de la vanguardia.

\section{El Corno Emplumado: ¿revista y movimiento?}

Si bien es cierto que célebres revistas de vanguardia resultan de la acción de un grupo concreto, como en el caso de Irradiador y Horizonte, las publicaciones del estridentismo mexicano, es cierto también que han existido revistas vanguardistas que, si bien no representan formalmente a un grupo, operan desde la conciencia de pertenecer u operar como representantes de un colectivo; recordemos por ejemplo la segunda época de la argentina Proa o las peruanas Amauta y Boletín Titikaka. En ese sentido, Pablo Rocca apunta que la noción de grupo es indivisible del proyecto de una revista cultural, pues es desde la colectividad que el proyecto estético que representa la revista se sostiene en el diálogo y debate con su entorno cultural, por ello expresa:

Hay una forma o célula básica de la revista: el grupo. ¿Hay otra forma posible que hacer una revista que no sea la de construir/se como grupo para que cada individuo sea, a la vez que sostén, complemento y puesta en crisis del otro, de los otros? (Rocca, 2004, p. 14)

Así pues, una revista representa en su materialidad un proyecto estético, pero también político cuando se articula como voluntad de interpelar a diversos actores sociales. Por ello, Beatriz Sarlo 
señala que la decisión de fundar una revista obedece a la certeza colectiva - manifestada gramaticalmente en el uso del "nosotros" - de que existe un vacío que es necesario llenar. Una revista, apunta, es una apuesta que busca transformar e incidir en un espacio social y cultural concreto, en suma, hacer una revista es hacer política cultural, por eso:

[...] "publiquemos una revista" quiere decir "hagamos política cultural", cortemos con el discurso el nudo de un debate estético o ideológico. La frase, cuya forma previsible es el plural, constituye el colectivo que suele quedar representado institucionalmente en una forma clásica: los consejos de dirección (Sarlo, 1991, p. 9, cursivas en el original).

La idea de la revista como un colectivo que se propone incidir en su realidad, coincide con la definición de movimiento que Renato Poggioli hace para referirse al proyecto vanguardista en su Teoría del arte de vanguardia. Al reconsiderar las agrupaciones artísticas a lo largo de la historia, Poggioli distingue entre escuela y movimiento, al destacar que mientras que la primera persigue fines exclusivamente artísticos, el movimiento "trasciende los confines de la literatura y el arte y se extiende a todas las esferas de la vida cultural y civil” (2011, p. 32). Por ello advierte:

Un movimiento se constituye ante todo para obtener un resultado positivo, un fin concreto. El resultado supremo deseado es, naturalmente, el éxito del movimiento específico o, en un plano más alto y más vasto, la afirmación del espíritu de vanguardia en todos los campos de la cultura y el arte" (p. 39).

De acuerdo con Poggioli, los movimientos de vanguardia se caracterizan primariamente por este ímpetu por transformar la vida desde el arte, en lo que él llama su momento "activista". Para Poggioli, el activismo vanguardista busca fundamentalmente el éxito del programa colectivo, pero, sobre todo, "cambiar en cualquier sentido el sistema social o político" (p. 41).

Este proyectarse desde los artístico al cuerpo social, es una práctica que en el caso de las vanguardias latinoamericanas adquiere especial relevancia como parte de un proceso que Vicky Unruh define como la "rehumanización" del arte, en referencia al concepto de José Ortega y Gasset: 
I would also argue more specifically that the drive toward engagement -intellectual, social, or metaphysical- was a defining feature of the international vanguard movements and that this was particularly true in Latin America (Unruh, 1994, p. 22).

[También diría más específicamente que el impulso hacia el compromiso -intelectual, social o metafísico- fue una característica definitoria de los movimientos internacionales de vanguardia y que esto fue particularmente cierto en América Latina]. ${ }^{2}$

Unruh sostiene que las vanguardias latinoamericanas asumen las empresas artísticas e intelectuales en términos de activismo y usan recursos usuales en el vanguardismo, como las técnicas antimiméticas, como vía para llevar al arte al terreno de la experiencia en términos más provocadores (1994, p. 22).

Por su parte, Katharina Niemeyer, al revisar la manera en que las vanguardias se insertan en los procesos sociales de la modernidad, explica que la práctica vanguardista, más que buscar una reconciliación entre el arte y la vida, presenta una cualidad compleja en la que coexisten, por un lado, su voluntad de emancipación como parte del proceso de autonomización del arte y, por otro, su voluntad de poder. Es decir que el ímpetu de las vanguardias busca al mismo tiempo mantener la autonomía del arte en relación con los otros campos del actuar social, a la vez que busca "ocupar desde esta base autónoma los otros ámbitos sociales" (2004, p. 28). A partir de esta posición, para Niemeyer, las vanguardias pueden considerarse bajo el concepto de "movimiento cultural" (2004, p. 28).

A partir de las nociones expuestas, sería entonces posible identificar en El Corno Emplumado atributos de un movimiento que busca constituirse como un factor de transformación no sólo del arte, sino también de la vida misma y del cuerpo social desde una acción artística de carácter colectivo. Para constatar este aserto, revisemos brevemente los orígenes y primeros pasos de la revista.

\footnotetext{
2 [Traducción propia]
} 
Es bien sabido que el impulso que da forma al Corno surge de las reuniones que jóvenes poetas y artistas tenían en el departamento del poeta beat Philip Lamantia en la Ciudad de México. En estas reuniones participaban poetas de México y Latinoamérica que leían sus poemas en español y poetas estadounidenses que leían sus poemas en inglés. Eran asiduos a las reuniones los mexicanos Homero Aridjis, Jaime Augusto Shelley y Juan Bañuelos; acudía también el nicaragüense Ernesto Cardenal, la poeta peruana Raquel Jodorowsky y el dibujante de origen salvadoreño, Carlos Coffeen Serpas. Otros artistas plásticos eran la pintora inglesa Leonora Carrington y el mexicano Felipe Ehrenberg, así como el poeta estadounidense Ray Bremser. Participaban, además, por supuesto, los futuros directores de la revista, Margaret Randall, Sergio Mondragón y Harvey Wolin, quien desistiría luego del segundo número (Silva, 2017, p. 55-56; Mondragón, 2006, s/p).

Según refiere la editora Margaret Randall, aunque entre el colectivo existía el sentimiento de una hermandad entre los poetas (citada en Silva, 2017, p. 14), pronto descubrieron que sólo se comprendían parcialmente ante la falta de buenas traducciones y que los poetas de habla española desconocían la tradición y actualidad de la poesía en lengua inglesa y viceversa, fue entonces que comenzó a fraguarse el proyecto de una revista (Randall 2015, p.101). A su vez, Sergio Mondragón destaca que durante las discusiones se formó rápidamente una dinámica de grupo que impulsó la creación de la revista:

En una de las sesiones, el grupo "descubrió" la necesidad, y "vio" en el azar que nos había reunido, la oportunidad de editar una revista que mostrara "los dos mundos" -el orbe de la poesía hispanoamericana y el de la poesía norteamericana: la poesía que se estaba escribiendo en ese momento.

$[\ldots]$

Se nombró encargados de la edición a Margaret Randall y Harvey Wolin -otro poeta beat que nos visitaba- por la parte norteamericana, y a Sergio Mondragón por la de español. Los fondos para el primer número, que apareció en enero de 1962, se reunieron en el curso de aquellas sesiones de lectura de poemas (Mondragón, citado en Willer, 2003, s/p). 
La declaración de Mondragón muestra que el proyecto tiene su origen en un colectivo no formalizado que impulsó la publicación. Así, la revista arranca en principio como medio para difundir la obra de ese grupo, de tal suerte que en el primer número aparecen varias de las autoras y autores que acudían a las reuniones, como Jaime Augusto Shelley, Carlos Coffeen Serpas, Ernesto Cardenal, Philip Lamantia, Leonora Carrington, Homero Aridjis y Raquel Jodorowsky, además aparecen textos de la editora Margaret Randall y los editores Sergio Mondragón y Harvey Wolin.

Sin embargo, la revista expande su horizonte rápidamente y el colectivo que la sustenta crece y se nutre de aportaciones de diferentes latitudes y ámbitos para ofrecer su respaldo no sólo moralmente, sino también financieramente, incluso en la venta y distribución de la revista. Los datos al respecto los proporciona la misma revista que en cada emisión incluía una lista de quienes aportaban recursos económicos, así como de sus representantes en diferentes partes del mundo. En esta red de apoyo solidario destaca, por ejemplo, la subasta que pintores mexicanos realizan para financiar la revista o la lectura que un grupo de mujeres poetas realizan en Nueva York, bajo el título "Cunts for Corno", con el fin de recaudar fondos para la revista (Mondragón 2006, s/p).

Vemos pues que El Corno Emplumado nace y se desarrolla como expresión de un colectivo de poetas y artistas. Adicionalmente, el hecho de que el Corno actúe como un movimiento cultural que postula como parte de su programa una transformación social desde la esfera estética, se hace evidente directamente en las páginas de la revista, pues cada número inicia con una nota de los editores en las cuales irán expresando los objetivos de la revista, mismos que exceden el ámbito puramente estético para proyectarse hacia la esfera de lo social. Así sucede desde el número inaugural, donde se anuncia la preocupación fundamental de la revista:

[...] ésta es una revista cuyas páginas están dedicadas a servir a la palabra y con las cuales se pretende crear la publicación que hoy hace falta... hoy día, cuando las relaciones entre los países de América son peores que nunca, esperamos que EL CORNO EMPLUMADO sea la mejor prueba (no política) de que TODOS SOMOS HERMANOS (El Corno Emplumado 1, enero 1962, p. 5. Cursivas en el original). 
De esta breve nota destacan varios puntos importantes para nuestra exposición. En primer lugar, como apuntaba Sarlo, existe la certeza de que una revista hace falta. En segundo lugar, se hace evidente que, como ocurre en la caracterización de movimiento de vanguardia de Poggioli, la revista se propone como un proyecto que desde el arte literario (la palabra) busca incidir en una realidad social (las relaciones entre los países de América). Finalmente, el breve mensaje proclama el carácter colectivo de la revista al hacer énfasis en la primera persona del plural (esperamos), lo cual será una constante en los textos editoriales de la revista. Tal sentido de comunidad será rematado al enunciar la convicción de la hermandad universal (todos somos hermanos).

Este sentido de movimiento estético-político que adquiere el Corno se irá construyendo al correr de los meses y años, hasta consolidar una red de articulaciones con otras revistas y colectivos que se formaliza en el Movimiento Nueva Solidaridad cuyo accionar cristaliza en el Primer Encuentro Americano de Poetas que se llevó a cabo sin apoyos oficiales los primeros días de febrero de 1964 y que contó con presencia de poetas de 15 países, según la notica que proporciona el propio Corno en su nota editorial del número 10. El encuentro fue organizado mayoritariamente por el Corno junto con la revista Pájaro Cascabel, también de México, y Eco Contemporáneo, de Argentina. Como fruto del encuentro, se difundió "La declaración de México" del Movimiento Nueva Solidaridad, en el que se expone el proyecto de una revolución espiritual encabezada por los artistas con miras a lograr la paz.

El documento aclara que esta revolución se origina en el arte, pero va mucho más allá para involucrarse con movimientos sociales, como la lucha por la defensa de los derechos civiles de la población negra en Estados Unidos o las luchas de emancipación de los pueblos aún colonizados y hasta las demandas por el desarme mundial. Para lograr esta revolución estética y política, el documento firmado por el Corno y reproducido en su número 10, puntualiza que la labor de las revistas es primordial:

Las revistas no son otra cosa que los signos externos de esa revolución interior, al igual que el resto de los sucesos en el campo político, científico y económico. Es importante decir que esta revolución es algo más que literaria [...] (El Corno Emplumado 10, abril 1964, p. 112) 
Esta convicción de que la revolución espiritual del arte necesariamente debía incidir en el devenir social, llevaría a la editora y al editor del Corno a no evadir su deber de conciencia y condenar desde sus páginas la creciente represión del gobierno mexicano contra el movimiento estudiantil de 1968, pronunciamiento que a la postre desencadenaría la persecución contra la revista y su consecuente desaparición:

El corno emplumado (sic) protesta enérgicamente contra semejante estado de cosas. Estamos conscientes de que los responsables de todo el problema son los jefes de Gobierno, quienes han demostrado su ineptitud, su crueldad y su ceguera espiritual. Y les decimos: esta violencia fue desencadenada por ustedes, quienes han usado a los jóvenes para sus movidas políticas; ha sido desencadenada por ustedes a través de toda una historia de mentiras, a través de la creación de este sistema que tiene como centro y dios absoluto al dinero, a través del pandillerismo internacional que sostiene al Gran Sistema y que se basa en la explotación y estupidización de los seres humanos, a través de la demagogia revolucionaria con que han gobernado nuestro país.

Los jóvenes estudiantes están, en el fondo, luchando contra este sistema corrupto. Ellos tienen en sus manos el futuro y el presente. La poesía y la vida es de ellos. Ustedes, viejos, no tienen nada que ofrecer. Ellos lo dan todo. Algunos de ellos ya ofrecieron su vida (EI Corno Emplumado 28, octubre 1968, p. 5-6).

\section{El umbral de la Nueva Era}

En la medida en que se ocupan en declarar los objetivos de la revista y el colectivo que representa, las notas editoriales que acompañan cada número del Corno funcionan a manera de los textos programáticos propios de las publicaciones de la vanguardia. Las notas editoriales dejan entrever la manera en que la idea de la poesía y el arte aparece y se va transformando a lo largo de los 31 números de la revista, pero también ofrecen pistas de la manera en que conciben la relación entre el arte, vida y política. 
Para nuestro análisis, estas notas editoriales son fundamentales para rastrear la discursividad que expresa la conciencia del umbral de época en que el Corno se ubica y cómo, desde esta conciencia, reivindicarán la necesaria transformación de la praxis vital desde el arte, en consonancia con el proyecto vanguardista. Por supuesto, la discursividad construida desde estas notas no está libre de contradicciones y ambigüedades, y aunque se irán transformando en su tono con el mismo discurrir y desarrollo de la revista en sus siete años de vida, sí es posible identificar ciertas constantes relativas a esa conciencia del umbral de época como momento de transición histórica de la cual el colectivo alrededor de la revista se asume como protagonista. Por ejemplo, en la nota editorial del segundo número se declara el advenimiento de tiempos inciertos frente a los cuales las determinaciones por parte del colectivo se hacen perentorias:

Nuestro órgano de difusión EL CORNO EMPLUMADO necesita de la ayuda de todos nosotros hermanos de la hora presente en la que se escuchan voces tan contradictorias y en la que los vientos presagian tormentas y tropiezos. Las máscaras están cayendo con sorprendente rapidez los acontecimientos angustian estamos en una paradoja la dolorosa condición del hombre se agudiza. Una cosa precisa el mundo: nuestra sinceridad y desprendimiento interior. Ello es la fraternidad. EL CORNO EMPLUMADO está abierto a todas las voces y todas las congojas (El Corno Emplumado 2, abril 1962, p.5).

De esta nota destaca en primer lugar que el punto de enunciación se ubica en un presente que mira hacia un futuro aciago e incierto. No obstante, la angustia que puede producir el oscuro porvenir, el texto constituye un llamado a la acción y formula con claridad su posición en este momento de cambio: la fraternidad universal como vía para liberar a las personas del sufrimiento.

Como ya se mencionó, cada número abre con una nota de los editores en español y en inglés, aunque regularmente ambas versiones coincidían, en muchas ocasiones presentaban diferencias. En el caso de la nota de los editores que abre el número dos, la versión en inglés ofrece una variante fundamental con respecto al texto en español, la cual se puede leer como una 
continuación de aquella y frente a la amenaza de ese futuro oscuro, se opone el esperanzador advenimiento de una nueva era cuyo signo debe ser la acción:

To lift the mask. The new era. Action undisturbed by reaction, up, down, left, right, inside - to the point from which is comes. And carried. Somehow to continue, giving what is being made, what is important and honest, carefully made

unmade:

"damned be the prism of the eye!"

(El Corno Emplumado 2, abril 1962, p.5)

[Abandonar las máscaras. La nueva era. Acción no perturbada por la reacción, arriba, abajo, izquierda, derecha, adentro, hasta el punto de donde surge. Y apunta. Una vía para continuar, para dar lo que ha sido dado, lo que es importante y honesto, hecho amorosamente

y deshecho:

“imaldito sea el prisma del ojo!"] ${ }^{3}$

A pesar de su tono críptico, se puede ver que las notas se adscriben a uno de los mitos de la modernidad, indisociable del proyecto vanguardista: el nuevo comienzo de la historia. En este punto, recordemos que Jauss coloca como uno de los más importantes mitos de la modernidad aquel que surge con la Revolución Francesa, cuando el hecho revolucionario es celebrado como el "cumplimiento del deseo de un nuevo comienzo de la historia, como acto fundamental de una sociedad de libres e iguales" (2004, p. 51). Este mito del nuevo comienzo toma forma concreta al instaurarse el calendario revolucionario que implica no sólo una nueva forma de medir el tiempo, sino el reinicio de la historia. Este ímpetu por instaurar una temporalidad revolucionaria fracasaría, hecho que sumado a la decadencia del legado intelectual de la llustración daría forma a los mitos del fin de la humanidad y la historia.

\footnotetext{
${ }^{3}$ [Traducción propia]
} 
Como corolario de este proceso, Jauss explica que, como producto de este desencanto, a partir de mediados del siglo XIX comienza a construirse la pretensión de que es necesario el fin de la historia para abrir un nuevo comienzo del mundo, aspiración que adquiere forma plena en las vanguardias. Así pues, al constatarse que desde la esfera de lo político se hace inviable un nuevo comienzo de la historia, entonces se postula "que sólo el arte puede suponer un nuevo comienzo del mundo, en un proceso aparentemente incontenible" (2004, p. 51). Desde la perspectiva de Jauss, el resultado de este proceso es el paso de las vanguardias del terreno artístico al político "para fijar en una revolución estética el nuevo comienzo de la historia bajo la dirección del arte", de ahí precisamente el origen de proclamas como las de futuristas como Balla y Depero que se arrogan la tarea de "construir de nuevo el Universo" (p. 62).

Esto explicaría porque en las notas editoriales del Corno es recurrente la presencia de este programa que postula el advenimiento de una nueva era de la humanidad, la cual sobrevendrá de la mano del arte. Sin embargo, el discurso de la revista no queda sólo en la forma testimonial, a modo de mera profecía, sino que se propone a sí misma como el agente desde donde esta renovación deberá surgir, siempre como expresión de una colectividad. Véase por ejemplo la nota editorial del número tres:

Montañas, árboles, ventanas, grandes edificios, niños, paisajes y la ciencia moderna; todas esas cosas las aceptamos abiertamente y sin sospecha. Es necesario que la expresión creativa de nuestro tiempo sea vista de la misma manera. Nuestra época -Cuba, África, Chessman, la bomba atómica, protestas colectivas, expresionismo abstracto, música electrónica, un millón de niños nacidos diariamente- reduce nuestra acción a una locura que fractura la luz en que nos movemos. Las respuestas que buscamos se nos ocultan tras la maquinaria, el dogma, los viejos odios y el funcionalismo de la sociedad. EL CORNO EMPLUMADO continuará sobre la base de que más allá de esas categorías estamos unidos por una fraternidad Ilamada arte (El Corno Emplumado 3, julio 1962, p. 5. Cursivas en el original). 
Aproximándose ya a su primer año, la misión del Corno se plantea como una labor de búsqueda y propuesta en ese espacio liminar e incierto. Esa locura que fractura las certezas se asume como esa grieta temporal entre lo que ya no es y lo que aún no es.

La conciencia de ubicarse en el umbral de una época para los jóvenes editores de El Corno Emplumado es el resultado del complejo contexto social que se vive internacionalmente desde la mitad de los años 50 del siglo XX. Aunque existe una cierta fe en la fraternidad a través del arte, los textos editoriales de la revista muestran la profunda angustia que la juventud de inicios de los años 60 experimenta en un entorno social amenazante.

Se viven aún los resabios del macartismo en Estados Unidos que desató una cacería de brujas contra cualquier atisbo de comunismo y disidencia. Con la expansión imperialista de Estados Unidos, en América Latina se suceden golpes de Estado y dictaduras militares en la región. A finales de los años 50 e inicio de los 60, la guerra fría entre los bloques alineados a Estados Unidos y la Unión Soviética entra en su momento más álgido y el temor a una conflagración nuclear cunde entre la juventud. No obstante, los procesos decoloniales en África y Asia, el triunfo de la Revolución Cuba y la incipiente lucha antirracista en Estados Unidos hacen renacer la esperanza de que un cambio es posible. En México, la situación es también compleja, pues el milagro mexicano revela su faceta autoritaria y el modelo del desarrollo estabilizador comienza a presentar sus fracturas, con lo cual se desatan oleadas de movilizaciones obreras con los ferrocarrileros y su líder, Demetrio Vallejo, al frente.

En medio de este caldo de cultivo, no es pues extraño que los jóvenes integrantes del colectivo alrededor del Corno sintieran la urgente necesidad de actuar, por ello, Margaret Randall, al recordar esos años destaca: “Esa sensación de desastre inminente (desesperación) contrarrestada por una apasionada visión poética (esperanza) permeaba cada uno de los números de la publicación" (2015, p. 114). ${ }^{4}$ Prueba de ello es el poema "Quetzalcoatl: 1961" que Randall publica

4 En cuanto a la manera compleja y contradictoria en que los agentes de las vanguardias enfrentan sus diferentes umbrales históricos, en los que la modernidad es a la vez promesa y amenaza, vale la pena recordar el análisis de Evodio Escalante del poema Urbe, del estridentista Manuel Maples Arce: “...este carácter oscilante es uno de los elementos que han dificultado a los críticos una correcta valoración de este poema maestro del estridentismo. Lo viejo y lo nuevo 
en el primer número del Corno, donde expresa: "even now/ your Mexico/ has become/ the last bomb shelter" (El Corno Emplumado 1, enero 1962, p. 14).

Si bien en el proyecto del Corno se hace presente la angustia ante un ambiente convulso, al mismo tiempo la publicación enarbola un activismo concreto que ve en el arte la única posibilidad de una renovación profunda basada en la poesía - de la misma manera en que Jauss apuntaba para la vanguardia-, por ello, en la nota editorial del número seis declaran:

Vivimos una nueva era, la Era del Hombre. Es nueva porque así lo han determinado procesos cósmicos, pero lo es también porque un hombre nuevo ha aparecido -y está apareciendo- en nosotros. Y los poetas, que son la voz de la tribu, cantan a este hombre nuevo; o mejor: desde este hombre nuevo.

Cantan con ritmo de salmo y palabras de aire, sencillos como el viento, confiados como el agua que sigue su curso. Cantan en todos los tonos, llenos de todos los colores y todas las esperanzas. A veces gritan como niños y sus gritos nos hieren los oídos del viejo hombre, pero todos cantan el solo acto de testimoniar lo nuevo.

EL CORNO EMPLUMADO es un instrumento para transmitir la nueva palabra, que, es decir, el nuevo espíritu. EL CORNO EMPLUMADO tiene su parte -pequeña y humilde- en este milagro.

EL CORNO EMPLUMADO saluda al Hombre de Aire

(El Corno Emplumado 6, abril 1963, p. 5).

En apoyo a este programa de acción ante el advenimiento de una nueva era, Mondragón y Randall recogerán en las páginas de la revista trabajos poéticos en los que precisamente resuena la

protagonizan una lucha que todavía no está decidida del todo, por más que el tema y el lenguaje del texto sorprendan por su actualidad al mismo tiempo maquínica y colectivista. La mayor paradoja es que el estridentismo se presenta como un movimiento progresista que, sin embargo, en un cierto nivel del texto, resiste el progresismo. Se abraza la modernidad al mismo tiempo que se le teme, así sea de modo inconsciente. Basta comparar el texto de Maples con los de Mayakovsky, o bien verlo a la luz del cine soviético soviético de la época (remito en particular a Lo nuevo y lo viejo de Einsenstein, de 1929), para advertir hasta qué punto el estridentismo mexicano no cree en el progreso a ciegas, ni de modo incondicionado: se advierte al mismo tiempo el triunfo de la tragedia. El futuro también es oscuro, y abre el abismo de la contingencia, para decirlo de otro modo. Es prometedor y a la vez catastrófico. Después de leer Urbe, uno queda convencido que el texto obedece a un proceso complejo de gestación..." (2002, pp. 53-54). 
necesidad de un cambio profundo en la sociedad y aparece el mito del nuevo comienzo, donde la unión fraternal será la norma. Un ejemplo es el poema "La ventana" de la ecuatoriana Ana María Iza:

\author{
Saldrá una nueva definición del hombre \\ en el gran diccionario de la vida. \\ Entrará la paz al universo \\ en un asno con la mirada limpia. \\ En fin: todos iremos juntos, \\ a través del sol y la neblina.
}

(El Corno Emplumado 6, abril 1963, p. 38)

En sentido similar, en el mismo número aparece el guatemalteco Otto-Raúl González con el poema "El águila encumbrada", donde se proclama también la proximidad de una era de renovación:

Soy la aurora. Soy el día

y la noche a un mismo tiempo.

[...]

Soy un hombre que la vida

socialista ha levantado

que vientos siderales

con estrépito saludan.

[...]

Soy mañana. Soy el pueblo.

Soy un águila encumbrada.

Soy el pan del habitante

de la tierra del futuro.

(El Corno Emplumado 6, abril 1963, p. 140-141)

Por supuesto es previsible que la selección de los poemas por parte de la editora y el editor se realizara buscando textos en consonancia con este programa porvenirista, sin embargo, hay 
elementos para suponer que se trataría de una idea que cunde generacionalmente y es compartida entre los colaboradores de la revista. En ese tenor destaca que la nota editorial del número siete reitere la idea de una nueva era donde se construya al nuevo hombre a la vez que se sugiera que la comunidad de poetas alrededor del Corno participa de este programa:

Un nuevo mundo se está elaborando, un tipo nuevo de hombre ya se perfila. Por todas partes surgen los signos.

\section{$[\ldots]$}

Y aunque la tormenta arrecia y las clavijas aprietan, los poetas siguen dedicados a la tarea de conocer y expresar su mundo interior. Los poetas, esos seres que se paran de pronto a escuchar el silbido del viento. Y el corno es su casa, y aquí están sus poemas de los últimos tres meses.

(El Corno Emplumado 7, julio de 1963, p. 5).

A manera de constatación de este programa, en el mismo número siete, en la selección de poesía cubana, se integra el poema "Y lo nuestro es la tierra" de Regino Pedroso, que augura:

Llegarán los grandes días

como monedas nuevas rodando sobre la vida,

y entonces nuestras manos se colmarán de júbilo!

[...]

Y lo nuestro

es la gran voz del viento,

que va segando en la mañana racimos de futuro!

(El Corno Emplumado 7, julio 1963, p. 43)

Para fortalecer el aserto de que la construcción de una nueva era es un programa compartido por la comunidad de poetas que confluyen en la revista, en el mismo número siete se reproducen una serie de cartas de los colaboradores donde manifiestan su convicción al respecto. Por ejemplo, en la 
misiva de Santiago Mathieu se deja entrever la misma percepción de ese estado liminar, en que el viejo orden está dejando de existir para dar paso a la renovación:

Está cercano el día se lo presiente se lo huele se lo puede oír galopar a lo lejos si colocamos nuestras orejas indias en el suelo en que todos habrán RESUCITADO en que se abrirán las tumbas y se oirán llantos de niños recién nacidos y se escucharán nuevos cantos y nuevas risas [...] (El Corno Emplumado 7, julio 1963, p. 172)

Antes de concluir, es necesario detenerse en la referencia al "Hombre de Aire", como el hombre nuevo que habrá de habitar la nueva era, pues se trata de una noción con profundas implicaciones en el proyecto de renovación que representa la revista.

Como ha explicado Gabriela Silva en su extenso análisis sobre El Corno Emplumado, la idea del hombre nuevo es una idea sintetizada como la posibilidad de que el individuo contemporáneo pueda convertirse en un ser superior a partir de una transformación profunda de su conciencia. Hacia los años 60, este tópico se propaga en Latinoamérica desde dos aspectos: por un lado, una visión espiritual y abstracta y, por el otro, una visión social y política, asumida por los grupos revolucionarios de la región (Silva, 2017, p. 145-146). ${ }^{5}$

La propia investigadora apunta que en el proyecto editorial del Corno confluyen las dos posturas: una más orientada a lo espiritual en los primeros años de la publicación y una más politizada hacia el final. Silva postula que el desarrollo de la revista ocurre en tres etapas que responden a las divergencias que las posturas estéticas y políticas de Randall y Mondragón adquieren progresivamente. En su examen, la autora destaca que la posición de la revista en cuanto a la incidencia del arte en la transformación social pasará de un planteamiento que idealiza la

\footnotetext{
5 La posibilidad idealizada del advenimiento del Hombre Nuevo fue una marca de época compartida por otros colectivos poéticos de México, por ejemplo, entre los integrantes del grupo La Espiga Amotinada, Juan Bañuelos, Óscar Oliva, Jaime Augusto Shelley, Jaime Labastida y Eraclio Zepeda, algunos de ellos también publicados en el Corno. Al respecto, puede verse el trabajo de Alí Hassan Rafael Franco, Hay que heredar la tierra, hermanos. El poeta y el hombre nuevo en La Espiga Amotinada. (2018).
} 
espiritualidad y el arte como vía de cambio social, a una postura que demanda intervenciones políticas concretas (2017, p. 139 y ss.). Así pues, la autora concluye que la primera etapa del Corno estaría marcada por una idea central: "el hombre contemporáneo es capaz de tomar consciencia plena de sí y de transformarse en un ser superior, en el sentido espiritual y humano de la expresión" (p. 145).

Desde su perspectiva espiritual y esotérica, inmersa en las ideas comunes a la contracultura de los años 60, los editores del Corno estaban convencidos de que la humanidad se encontraba en el umbral entre dos eras astrológicas: la era de Piscis que llegaba a su fin, para dar paso a la era de Acuario, la cual se anunciaba como la gran era de las trasformaciones. Al respecto, el propio Mondragón escribía a su colega y amigo Miguel Grinberg, editor de la revista argentina Eco Contemporáneo:

[...] astrólogos tibetanos han anunciado el fin de la era de Piscis, y el comienzo de la era de Acuario, una era de despertar, lo cual traducido a nuestro lenguaje occidental sería más o menos la era del hombre (Mondragón, citado en Manzano, 2017, p. 122).

Esta convicción de ubicarse en el periodo del cambio de la era astrológica la reiterarán los editores en la nota de presentación del número nueve de la revista, que reza:

En ocho números hemos publicado poesía, prosa, teatro, cartas, arte, y hablado acerca de una Nueva Era habitada por un Hombre Nuevo. Muchos preguntan todavía quién es este hombre nuevo y en qué nueva era vive. Uno puede inclusive sentir el cambio dentro de sí y resistirse -como nosotros a veces - a ponerle un nombre. Piscis/Acuarius. Una revolución espiritual que se compara históricamente con la revolución industrial: un salto desde la máquina hasta la mente y el corazón. (El Corno Emplumado 9, enero 1964, p. 5)

El signo astrológico de Acuario, recordemos, a pesar de su nombre corresponde precisamente al elemento aire, pero según refiere el Diccionario de los símbolos, su "resonancia acuática da testimonio de una sustancia nutritiva más destinada a apagar la sed del alma que la del cuerpo", es un signo que "plantea el mundo de las afinidades electivas, que nos convierten en seres que viven 
en una comunidad espiritual y en plena esfera universal" (Chevalier y Gheerbrant, 1993, p. 48). Incluso, explica el mismo diccionario, existe también un Acuario uránico y prometeico, que es "el ser de la vanguardia, del progreso, de la emancipación, de la aventura" (p. 48).

En suma, desde la perspectiva de la revista, el hombre nuevo es el hombre de aire, de la era de Acuario, era de la transformación del Espíritu y de la fraternidad, el hombre de la vanguardia, de la innovación y la búsqueda libertaria. Esta dimensión esotérica adquiere en las páginas del Corno un impacto decisivo que incide tanto en aspectos temáticos como formales de sus contenidos.

En primer lugar, es evidente que el mismo título de la revista contiene este aspecto trascendente del aliento espiritual en la figura del corno o trompeta, la cual por sí misma contiene diversas reminiscencias vinculadas al anuncio de los grandes acontecimientos históricos y cósmicos (Chevalier y Gheerbrant, 1993, p. 1027). En cuanto a la génesis del nombre de la revista, Randall y Mondragón han referido que con él buscaban sintetizar por un lado el mundo del jazz, con toda su libertad creativa y su filiación a la contracultura y, por otro, la potencia de las culturas prehispánicas encarnadas en el mito de Quetzalcóatl, la serpiente emplumada (Randall, 2015, p. 102).

Esta idea del aliento primordial que alimenta la música y la libertad expresiva propia de la improvisación del jazz, más allá de la mera ocurrencia, se trata de un paradigma que tuvo gran difusión entre la poesía joven del continente, de tal suerte que llegó a constituir un paradigma de innovación formal de los principios de composición de la época.

En ese sentido, Gabriel Ramos (2018) señala que de la mano de las exploraciones que los poetas Beats habían realizado en la década de los 50, en cuanto a la conceptualización de la poesía como aliento vital y musical, como canto determinado por el ritmo del flujo inspiración-exhalación, se difunde entre los jóvenes poetas latinoamericanos el uso del verso libre, sin restricciones ni en la forma ni en su sentido, ya que:

La forma se confirma en un discurso libre, motivado por un ritmo vital común al individuo, a la naturaleza y a la historia. El poema es el registro de la búsqueda de las imágenes, no su conclusión o condensación; sin una ordenación restrictiva, aparece a veces prolijo (2018, p. 18). 
El mismo Ramos refiere que fiel al espíritu de la época, en el Corno aparecen poemas que responden a este principio compositivo y cita como ejemplo el poema "Occidental saxo" de Jaime Augusto Shelley, publicado en el primer número, donde se lee:

Edgar Blackie, contrabajo negro

Arriba

sube los peldaños

de una escalera interminable en busca de trabajo

[...]

Edgar baja sube infinitos peldaños

escaleras desanda pasillos y ascensores

tropezando siempre con su risa a cinco dedos

$[\ldots]$

Mr. Blackie músico y viajante

tañedor del siglo XX.

(Shelley citado en Ramos, 2018, p. 23)

En cuanto al sentido cósmico-espiritual que la noción del hombre de aire adquiere en las páginas de la revista, la propia Margaret Randall publica en el número uno de la revista, el poema "The joining of the sign" -quizá en referencia al signo de Acuario-, en el cual el aire se asocia al espíritu como pneuma o aliento vital que insufla de conciencia despierta a la voz enunciativa, en una aparente reminiscencia de las prácticas religiosas de Oriente:

great spirit descending descending

where

why where battering

web of complications silence of complications / to

breathe away a wa y

glorious (they know) glorious 
in quiet places

filling / casting off of before

the questions

and filling time.

(El Corno Emplumado 1, enero 1961, p. 12)

[El gran espíritu desciende desciende

dónde

por qué, dónde palpita

en la red de los dilemas en el silencio del dilema / para

respirar exhalar ex $\mathrm{h}$ a I a r

y es glorioso (lo saben) es glorioso

en el espacio y el silencio

es pleno / y en el origen expulsa

las dudas

plenitud del tiempo $]^{6}$

El poema propone además un juego de símbolos, en el que el aire se asimila a la luz solar, en su aspecto de fuerza genésica, a la vez que potencia divina (aire-luz) que ilumina y expande la conciencia:

happening in the sun

light / air full of sun sun

full of air

here happening in brightness

the sun

when before a womb of rain

was web of consciousness

(El Corno Emplumado 1, enero 1961, p. 13)

\footnotetext{
${ }^{6}$ [Traducción propia]
} 
[Acontence en el sol

luz / aire pleno de sol sol

pleno de aire

aquí emerge el brillo

el sol

así, ante el vientre de la lluvia

fue lienzo para la conciencia] $]^{7}$

En cuanto a su forma, el poema destaca por intercalar versos amplios con otros muy breves que tanto musicalmente, como gráficamente replican el ritmo pausado de la respiración controlada de las prácticas de meditación.

El tema del aliento/respiración/música/canto aparece también en la selección de poemas que el otro editor, Harvey Wolin, que se incluyen en el número inaugural del Corno. Al igual que los textos de Randall, los poemas de Wolin se organizan en versos irregulares y singulares juegos de paronomasias para aludir a la respiración, por ejemplo, en el poema "Momma blues", cuya musicalidad está obviada en su título:

im (sic) everything in the morning

nothing by time of night

i hear my vespered breathing

whisper Sister Whisper

repeat the rhyme of blood

(El Corno Emplumado 1, enero 1961, p. 38)

[soy totalidad matutina

nada soy en la hora nocturna

\footnotetext{
7 [Traducción propia]
} 
escucho mi aliento vesperal susurra Hermana Susurrante

repite la rima de la sangre $]^{8}$

Por su parte, Sergio Mondragón presenta una serie de dos poemas, "Retablo primero" y otro sin título, en el primero de los cuales cuestiona la posibilidad de conocimiento desde la racionalidad occidental, ejemplificada en el número Pi, mientras que en el segundo aparece de nueva cuenta el tema del aliento, caracterizado aquí como posible respuesta a una búsqueda vital que se antoja infructuosa:

\author{
busco tu rostro en los árboles \\ busco tus ojos en la obscuridad \\ de mis noches de fiebre \\ encuentro tu aliento. \\ tomo tu sangre.
}

(El Corno Emplumado 1, enero 1961, p. 80)

\title{
Conclusiones
}

Al final de la década de 1950 el mundo vivía una serie de reacomodos en la política y la economía, sombras aciagas se elevan en el horizonte a la vez que surgen las evidencias de que una transformación social es viable. En palabras de Jauss, estamos ante un umbral de época: la situación es la de un mundo que no acaba y un nuevo horizonte que aún no es. En este contexto surge la revista El Corno Emplumado/The Plumed Horn como empresa de un grupo de jóvenes poetas y artistas en su proceso de tomar conciencia de la necesidad de ser protagonistas de ese umbral histórico.

Como parte de este proceso, El Corno Emplumado actualiza una serie de actitudes y procedimientos asimilados a las vanguardias. En principio, la creación de la revista resulta del

${ }^{8}$ [Traducción propia] 
encuentro de un colectivo que recupera el impulso utópico que ubica en el arte la reformulación de una praxis vital que incida en la transformación social, donde la poesía deberá ser el paradigma y eje de referencia. A fin de asumirse como actor de este umbral de época que debería desembocar en la transformación social, El Corno Emplumado actualiza el mito vanguardista del nuevo comienzo y (re)construye discursivamente la utopía del inminente advenimiento de una Nueva Era, la era del Hombre Nuevo, el Hombre de Aire, como una era de revolución estética y espiritual que traerá consigo la fraternidad universal. Una vez consolidado como proyecto editorial, El Corno Emplumado irá fortaleciendo sus vínculos, sumando simpatías y apoyos para configurar un auténtico movimiento cultural que mantiene en su horizonte la tarea primara de transformar la vida desde el arte.

En suma, la longeva empresa editorial del Corno es una evidencia de que para los años 60 ciertos elementos del proyecto vanguardista perviven en el horizonte social como una práctica viable que ofrece las bases conceptuales y discursivas para hacer frente a una realidad que exige de los artistas una toma de posición. Sergio Mondragón y Margaret Randall logran dar forma a las manifestaciones y actitudes de un amplio colectivo que, aún diverso y heterogéneo, comparte la visión de que una transformación es necesaria y posible. Desde este punto de vista, puede postularse que, en cuanto movimiento cultural de carácter estético y político, el Corno significa un elemento de continuidad del proyecto vanguardista, actualizado y transformado para adecuarse a la realidad específica que los jóvenes de los años 60 enfrentan.

La actitud vanguardista de desconfianza hacia la razón instrumental, se replica en el programa del Corno como rechazo a la ciencia occidental, percibida como la progenitora de las armas nucleares. Por ello, las búsquedas de los jóvenes poetas agrupados en la revista los llevan a buscar saberes alternativos y exploran en las religiones orientales, el esoterismo, la astrología y el tarot. Estos elementos, como vimos, moldean el discurso programático de la revista e inciden en aspectos formales y temáticos de las obras que publican.

Aunque en las páginas del mismo Corno no se manifiesta explícitamente una intención o interés de asumirse como una continuidad de la vanguardia, se puede apreciar que cuando un 
maduro Sergio Mondragón revisa en retrospectiva la juvenil revista, entiende que la publicación operó en el contexto de lo que podemos llamar un continuum vanguardista.

En ese sentido, recordemos que en 1988 Mondragón escribe el prólogo para la primera edición de Poemas estridentistas de Germán List Arzubide, donde apunta que el impulso vanguardista, representado en México por el estridentismo, continuó nutriendo a una parte importante de la poesía mexicana, la más experimental, la cual se hace más visible en los años 60 , misma que se difunde en revistas independientes como Pájaro Cascabel, Cuadernos del Viento, Mester, El Rehilete y, por supuesto, El Corno Emplumado. Gracias a esta persistencia vanguardista, apunta Mondragón en el mismo prólogo, se ha construido "una genealogía que se prolonga hasta el día de hoy, en los poetas más jóvenes, aquellos que hacen de la volatilidad y asimetría de su lenguaje la aventura por excelencia de sus vidas" (1998, p. 21).

En estas palabras de Mondragón se infiere su convicción de que el impulso vanguardista no se extingue en un momento determinado de la historia, sino que se trata de una potencia capaz de pervivir en cada actualidad, en forma de oleadas, como transformaciones permanentes, a la manera apuntada por Jauss y Del Gizzo, y como el propio editor mencionaba en su citada hipótesis de una vanguardia permanente.

Aunque es usual la idea de identificar el quehacer de las primeras vanguardias como un episodio efímero, existieron algunos de sus protagonistas que visualizaron su proyecto de transformación como una tarea que, para concretarse, necesariamente debía prolongarse en el tiempo. Muestra de ello es el pensamiento de José Carlos Mariátegui, que para el segundo aniversario de Amauta -revista indispensable para la vanguardia latinoamericana- luego de haberse suspendido la edición de la misma, aclaraba:

Pero Amauta no había nacido para quedarse en episodio, sino para ser historia y para hacerla. Si la historia es creación de los hombres y las ideas, podemos encarar con esperanza el porvenir. De hombres y de ideas, es nuestra fuerza.

La primera obligación de toda obra, del género de la que Amauta se ha impuesto, es ésta: durar. La historia es duración. No vale el grito aislado, por muy largo que sea su eco; vale la prédica 
constante, continua, persistente. (Amauta 17, septiembre 1928, p. 1. Reproducido en Müller-Bergh y Mendoça, 2005, p. 175)

Finalmente, queda claro que, con la perspectiva de los años, el editor de El Corno Emplumado identifica a su revista como protagonista de este continuo vanguardista. Tal formulación abre nuevas interrogantes en cuanto a las particularidades y componentes de esta hipotética "vanguardia permanente" y sobre cuál sería el papel de El Corno Emplumado en esta articulación y, sobre todo, cómo participarían de esta serie los agentes colectivos asociados a la idea de vanguardia que anteceden al Corno y los que aparecen posteriormente, como el grupo poeticista, el colectivo de La Espiga Amotinada y el movimiento infrarrealista.

\section{Referencias}

Chevalier, J. y Gheerbrant, A. (1993). Diccionario de los símbolos, Barcelona: Herder. Del Gizzo, L. (2015). La vanguardia después de la vanguardia. El invencionismo y su deriva en el movimiento poesía buenos aires (1944-1963), tesis doctoral, Buenos Aires: Universidad de Buenos Aires, Facultad de Filosofía y Letras.

Escalante, E. (2002). Elevación y caída del estridentismo. México: Consejo Nacional para la Cultura y las Artes/ Ediciones Sin Nombre.

Franco, A. (2018), Hay que heredar la tierra, hermanos. El poeta y el hombre nuevo en La Espiga Amotinada, tesis. México: Universidad Autónoma Metropolitana- Azcapotzalco/ Especialización en Literatura Mexicana del Siglo XX.

Galindo, Ó. (2013). Neovanguardias hipervitalistas en la poesía hispanoamericana. Taller de Letras, (52), 1-37.

Jauss, $\mathrm{H}$, (2004). Las transformaciones de lo moderno. Estudios sobre las etapas de la modernidad estética, (traducción de Ricardo Sánchez Ortiz de Urbina), segunda edición, Madrid: Antonio Machado Libros. 
Manzano, V. (2017). Fraternalmente americanos: el Movimiento Nueva Solidaridad y la emergencia de una contracultura en la década de 1960. Iberoamericana América Latina - España Portugal, 17, (66), 115-138.

Mondragón, S. (1998). “La poesía como aventura”, en Germán List Arzubide, Poemas estridentistas. México: El Tucán de Virginia.

Mondragón, S. (2006). El Corno Emplumado. Una revista mexicana de poesía con una vocación latinoamericana. Alforja, (36), s/p. Versión digital. Obtenida el 29 de marzo 2021 de: https://fdocuments.mx/document/ver-revista-pdf.html>

Müller-Bergh, K. y Mendoça Teles, G. (2005). Vanguardia Latinoamericana. Historia, crítica y documentos, vol. IV,. Madrid/Frankfurt am Main: Iberoamericana/Vervuert.

Niemeyer, K. (2004). Subway de los sueños, alucinamiento, libro abierto. La novela vanguardista hispanoamericana. Madrid/Frankfurt, Iberoamericana-Vervuert.

Premat, J. (2013). Los relatos de la vanguardia o el retorno de lo nuevo. Cuadernos de Literatura, (34), 47-64.

Poggioli, R. 2011.) Teoría del arte de vanguardia. México, UNAM.

Ramos, G. (2018). “Alternativas de la forma libre en la poesía mexicana de la década de 1960”, Tema y Variaciones de Literatura (50), 13-35.

Randall, M. (2015). Recordando El Corno Emplumado, Revista Casa de las Américas (280), 100-118.

Rocca, P. (2004). Por qué, para qué una revista (Sobre su naturaleza y su función en el campo cultural latinoamericano). Hispamérica, (99). 3-19.

Sarlo, B. (1991). Intelectuales y revistas, razones de una práctica. América: Cahiers du CRICCAL. (9). 109-16.

Silva, G. (2017). Texto, contexto e índices de El Corno Emplumado (1962-1969), tesis, México: El Colegio de San Luis, Maestría en Literatura Hispanoamericana.

Unruh, V. (1994). Latin American vanguards: the Art of Contentious Encounters. Berkeley: University of California. 
Willer, C. (2003). El Corno Emplumado e Eco Contemporáneo, grandes momentos da historia da cultura Ibero-Americana: Sergio Mondragón e Miguel Grinberg. Agulha. Revista de Cultura, (32). s/p. Obtenida el 29 de marzo 2021 de:

http://www.jornaldepoesia.jor.br/ag32willer.htm. 